\title{
Ecritures et culture digitale
}

\section{$1 \quad$ Préambule}

Cet essai souhaite démontrer que l'écriture digitale contribue à l'émergence « d'un nouveau rapport du corps de l'homme aux machines », tel qu'annoncé par Jacques Derrida lorsqu'il commente les effets à venir des nouvelles technologies ${ }^{1}$. Ces liens contractés par l'écriture à la matière digitale et aux corps convient en retour la tradition judéo-chrétienne à réfléchir à leurs conséquences pour ce corpus textuel désigné souvent comme « les Ecritures », soit la Bible.

Ce livre s'attache donc à scruter le devenir de l'écriture digitale et des Ecritures digitales, deux parcours rassemblés en français en une seule expression qui sert de titre : Ecritures digitales. Le sous-titre anglais l'explicite :Digital writing, digital Scriptures. Le rôle de cette autre langue est pleinement assumé dans ces pages comme partenaire scientifique avec lequel la recherche francophone en sciences humaines doit désormais compter. Chaque chapitre sera résumé en anglais.

Après une introduction générale, deux chapitres couvrent la thématique de l'écriture digitale (chapitres 2 et 3), encadrés par deux chapitres sur les Ecritures digitales (chapitres 1 et 4 ). Les chapitres 1 et 2 conduisent une analyse générale de la problématique et des notions clés, alors que les chapitres 3 et 4 mènent l'enquête à partir de l'hypothèse de départ, pour chacune des deux thématiques. La conclusion la vérifie, en résumant les points principaux de l'itinéraire.

\section{$2 \quad$ Ecritures en jeu}

En 2009, Jean-Claude Carrière et Umberto Eco exprimaient toute leur passion pour le livre dans un ouvrage au titre éloquent, N'espérez pas vous débarrasser des livres, né de leurs entretiens avec Jean-Philippe de Tonnac. Ce dernier déclare au début de la préface :

1 J. Derrida, Sur parole. Instantanés philosophiques, Editions de l'Aube, 200o, édition Kindle, l. 484 ; citation complète p. 3 ci-dessous. 
Nos «Bibles de pierre », [les cathédrales], n'ont pas disparu, mais l'ensemble de la production des textes manuscrits, puis imprimés, cette « fourmilière » des intelligences, cette « ruche où toutes les imaginations, ces abeilles dorées, arrivent avec leur miel » les a soudain, à la fin du Moyen-Âge, singulièrement déclassées. De la même manière, si le livre électronique finit par s'imposer aux dépens du livre imprimé, il y a peu de raisons qu'il parvienne à le faire sortir de nos maisons et de nos habitudes. L'« ebook» ne tuera donc pas le livre. Pas davantage que Gutenberg et sa géniale invention n'ont supprimé du jour au lendemain l'usage des codex, ni celui-ci le commerce des rouleaux de papyrus ou volumina ${ }^{2}$.

Dans ces quelques lignes se trouvent croisés les thèmes de l'écriture - manuscrite, imprimée et électronique -, du livre, et de la Bible. Jean-Claude Carrière accentue leur interaction en se risquant même à déclarer plus loin qu' « avec les religions du Livre, le livre a servi non seulement de contenant, de réceptacle, mais aussi de 'grand angle' à partir duquel on pouvait tout observer et tout raconter, peut-être même tout décider $»^{3}$. Quand bien même on resterait prudent devant l'emphase rhétorique de cet énoncé, il est difficile de nier que la Bible judéo-chrétienne, cet « objet livre », a pu fonctionner ainsi - et fonctionne encore - dans la culture occidentale et au-delà. On pourra en prendre comme simple exemple la porte d'accueil monumentale qui attendait les visiteurs de l'exposition internationale organisée en l'honneur des 500 ans de la Réforme, à Wittenberg, en mai 2017 : elle consistait en une Bible de vingt-sept mètres de haut ${ }^{4}$, porte de salut ou fourches caudines à franchir, selon les sentiments de chacun. Les liens entre matérialité du livre, concept de « religions du livre » et autorité seront abordés au chapitre 4 de cet essai.

Dans l'immédiat, les propos de Jean-Claude Carrière signalent que vouloir en découdre avec l'écriture électronique s'émancipant du livre, signifie faire face à la symbolique culturelle de la Bible dans son lien au livre. Un tel constat ne peut qu'interpeler les biblistes, dont je suis, à réfléchir à ce qu'il advient des «Ecritures» sacrées pour la tradition judéo-chrétienne dans la culture digitale. Umberto Eco, quant à lui, évoquant de mille façons dans N'espérez pas son attachement au livre, ouvre à d'autres problématiques encore. Il décrit notamment l'écriture manuelle comme une technique «presque biologique » qu'il distingue par cette caractéristique des technologies électroniques :

2 J.-P. de Tonnac, «Préface », dans J.-C. Carrière - U. Eco, N'espérez pas vous débarrasser des livres, Seuil, 2009, édition Kindle, l. 39-45.

3 Carrière - Eco, N'espérez pas, édition Kindle, l. 1290-1298.

4 Voir Reformation 2017, <https://r2017.org/weltausstellung/welcome/>. Tous les liens mentionnés dans ce livre ont été vérifiés pour la dernière fois le 18.04.19. 
Nous pouvons considérer que l'écriture est le prolongement de la main et dans ce sens elle est presque biologique. Elle est la technologie de communication immédiatement liée au corps. Lorsque vous avez inventé cela, vous ne pouvez plus y renoncer. [...] Tandis que nos inventions modernes, cinéma, radio, Internet ne sont pas biologiques ${ }^{5}$.

Dans cette citation, l'écriture est encore forcément comprise comme l'écriture manuelle : elle est considérée comme le prolongement de la main. On comprend implicitement qu'Eco n'aurait pas nommé ici écriture ce qu'il produit via le support digital, fût-ce de ses doigts sur l'écran d'un smartphone. De surcroît, dans ce discours publié en 2009, le lien au biologique apparaît comme un argument supplétif chez Eco, alors que la problématique est devenue aujourd'hui un point clé de l'évolution des nouvelles technologies : qui ne s'étonnerait pas devant les innombrables avancées des bio-technologies qui précisément unissent chaque jour davantage notre corps aux techniques, telle la jambe bionique, annoncée au printemps $2015^{6}$ ? Au contraire d'Umberto Eco, douze ans auparavant, Jacques Derrida décrivait déjà le tournant technologique comme impactant le corps :

Ce qui se prépare, à un rythme encore incalculable, de façon à la fois très lente et très rapide, c'est un nouvel homme bien sûr, un nouveau corps de l'homme, un nouveau rapport du corps de l'homme aux machines, et on l'aperçoit déjà cette sorte de transformation 7 .

Le rythme est désigné ici comme un paramètre capital, mais inconnu de la transformation en cours : le chapitre 3 sera attentif à la notion du rythme et à son incidence sur l'écriture et la production de la connaissance. Ce n'est pas à la légère que Derrida proclame le «nouveau rapport du corps de l'homme aux machines ». Dans un article de 1997, il avait longuement décrit la sortie du papier comme un « séisme » $\operatorname{corporel}^{8}$ : « Depuis que j'ai commencé à écrire, le

5 Carrière - Eco, N'espérez pas, édition Kindle, l. 197-203.

6 Voir Brain-Controlled Bionic Legs Are Finally Here, <https://www.popsci.com/brain-con trolled-bionic-legs-are-here-no-really>.

7 Derrida, Sur parole, édition Kindle, l. 484; signalé en page 1, note 1 ; version orale: C. Paoletti - B. Sourcis, « Jacques Derrida. A voix nue » 4/5, interview radiophonique réalisé en 1998 et rediffusé sur France Inter le 14.10.2010 et le 29.01.2016, 29 minutes ; citation à $16 \mathrm{~min} 49$; <https://www.franceculture.fr/dossiers/jacques-derrida-l-integrale-en-cinqentretiens-1998>.

8 Voir J. Derrida, «Le papier et moi, vous savez.... », Les Cahiers de médiologie 4 (1997), p. 33-57; <https://www.cairn.info/revue-les-cahiers-de-mediologie-1997-2-page-33. htm\#> ; version anglaise : J. Derrida, « Paper or me, You Know... (New Speculations on a 
statut et la stabilité du papier ont été constamment secoués par des secousses sismiques [annonçant] la 'perte' d'un support : la fin du 'subjectile' approche. C'est aussi par là, sans doute, que ce corps de papier nous tient au corps $»^{9}$.

Contrairement à Umberto Eco qui ne considère pas encore en 2009 le possible retrait ou repli partiel de l'écriture manuelle, Derrida, douze ans auparavant, fait face à l'« angoisse » qui serait provoquée « par le retrait de l'écriture acérée à la pointe d'une plume sur une surface de papier, le retrait d'une main, d'un certain usage de la main unique en tout cas $\gg^{10}$. Cette angoisse s'est de fait concrétisée dès 2015 par des annonces précipitées dans la presse francophone de fin de l'apprentissage de l'écriture manuelle ${ }^{11}$ : il s'agissait en fait d'annonces venues des Etats-Unis et de la Finlande où le choix avait été fait d'arrêter l'enseignement non pas de l'écriture manuelle mais de l'écriture cursive, pour se centrer sur l'apprentissage de l'écriture en lettres détachées. Dans les pays concernés, plusieurs voix se sont bien sûr élevées pour dire l'importance inaliénable de l'apprentissage de l'écriture manuelle ${ }^{12}$.

Par-delà ces phénomènes d'angoisse liés à une surenchère médiatique, l'impact des nouvelles technologies sur notre corporéité ne fait plus de doute, ne serait-ce qu'en écrivant sur écran du bout de nos doigts. Un article de $2014 \mathrm{pu}-$ blié dans Current Biology démontre l'existence de nouvelles stimulations et l'impact (positif) sur le cerveau de l'usage des doigts et pouces, via les smartphones $^{13}$. C'est la confirmation physique, biologique, de ce que Robert Darnton, président des bibliothèques de Harvard, avait désigné en 2009 comme la découverte d'un monde nouveau avec ses doigts, si bien décrite en allemand par le sentiment de Fingerspitzengefühl:

Luxury of the Poor)», dans J. Derrida, Paper Machine, the Board of Trustees (trans.), Stanford University Press, $2005^{2}$, p. 41-65; <https://www.scribd.com/document/292219 602/Derrida-Paper-or-Me-You-Know-from-Paper-Machine>.

$9 \quad$ Derrida, « Le papier et moi », p. 34.

10 Derrida, « Le papier et moi », p. 46.

11 C. Clivaz, « Covers and Corpus wanted! Some Digital Humanities Fragments », DHQ 10/16 (2016), §13, <http://www.digitalhumanities.org/dhq/vol/10/3/000257/000257.html> ; version française: C. Clivaz, «En quête des couvertures et corpus. Quelques éclats d'humanités digitales », dans V. Carayol - F. Morandi (éd.), Le tournant numérique des sciences humaines et sociales, MSHA, 2015, p. 97-109.

Voir par exemple P. Klass, «Why Handwriting Is Still Essential in the Keyboard Age », New York Times Blogs 24 juin 2016, <https://well.blogs.nytimes.com/2016/06/2o/whyhandwriting-is-still-essential-in-the-keyboard-age/>.

A.-D. Gindrat et al., « Use-Dependent Cortical Processing from Fingertips in Touchscreen Phone Users », Current Biology (23.12.2014), DOI : <http://dx.doi.org/10.1016/j.cub.2014.11. o26> ; cité dans C. Clivaz, « De 'numérique' à 'digital' », Le texte à venir. Etudes digitales 1 (2016), p. $255^{-256}$; ici p. 256. 
Nous trouvons notre chemin dans le monde par les moyens de la disposition sensorielle que les Allemands nomment Fingerspitzengefühl. Si vous aviez l'habitude de guider un stylo avec votre index, prêtez attention à la manière dont les jeunes utilisent leurs pouces sur les téléphones mobiles, et vous verrez combien la technologie pénètre une nouvelle génération, corps et âme ${ }^{14}$.

A relire Derrida et Darnton, on est invité à réfléchir au retrait - au moins partiel - du papier, qui pousse l'écriture à un nouveau mode de relation avec le corporel, par l'intermédiaire de technologies numériques. Un tel constat ne peut qu'inciter les chercheurs en sciences humaines à réfléchir à ce que devient l'écriture dans la culture digitale, du papier au support numérique, de l'écriture manuelle à l'écriture électronique. Dans cette réflexion s'est invité le corps, et il sera un thème récurrent de cet ouvrage. Le chapitre 2 mettra notamment en évidence les sens oubliés du mot désuet « humanités » - sens oubliés qui sont précisément le corps, la chair. Le pluriel d'« humanités » est un mot revenu sur le devant de la scène via la traduction française de l'expression anglaise digital humanities par «humanités numériques » ou « humanités digitales », une double traduction qui signale l'importance plus forte que jamais de la langue et des mots.

\section{La langue et les mots}

Le propos du présent ouvrage est donc de s'interroger à la fois sur l'écriture, ce savoir-faire profondément ancré au cœur des sciences humaines, et sur les Ecritures bibliques. Le titre de cet ouvrage en français, Ecritures digitales, implique ces deux sens dans le terme « écritures », la majuscule initiale masquant toute possibilité de les distinguer. Mais la langue anglaise l'explicite dans le soustitre: Digital writing, digital Scriptures. Ces titre et sous-titre en deux langues s'étayent l'un l'autre, soit par la synthèse soit par l'explicitation, et expriment

14 R. Darnton, The Case for Books: Past, Present, Future, Public Affairs, 2009, p. XIII : « we find our way through the world by means of a sensory disposition that the Germans call Fingerspitzengefühl. If you were trained to guide a pen with your finger index, look at the way young people use their thumbs on mobile phones, and you will see how technology penetrates a new generation, body and soul »; cité dans C. Clivaz, « Common Era 2.o. Mapping the Digital Era from Antiquity and Modernity», dans C. Clivaz - J. Meizoz F. Vallotton - J. Verheyden (éd.), avec B. Bertho, Reading Tomorrow. From Ancient Manuscripts to the Digital Era / Lire Demain. Des manuscrits antiques à l'ère digitale, P PUR, 2012, ebook, p. 23-60 ; ici p. 27. 
l'une des modifications à l'œuvre dans les humanités en voie de digitalisation : le rapport à la nouvelle lingua franca, l'anglais, est devenu partie prenante de la production du savoir dans les sciences humaines, qu'on s'en réjouisse ou le déplore. L'anglais, ou plutôt faut-il dire le globish, selon un terme commenté dans un article de Marin Dacos ${ }^{15}$ :

La communauté des humanités numériques est anglophone en raison de la large diffusion de l'enseignement de l'anglais dans le monde, et non parce que les anglophones de naissance sont numériquement dominants dans cette communauté. Acceptons donc l'anglais comme seconde langue. En tant que seconde langue, il sera le Globish, et non l'English. Ce globish, mécaniquement plus rustique et rudimentaire que l'anglais d'Angleterre, sera un véhicule de communication entre égaux. [...] Audelà de la maîtrise technique, c'est de confiance en soi, de confiance globish, que nous devons doter notre communauté. Sinon, comment expliquer que nous ayons si peu de candidats globophones dans les élections de nos organisations? C'est bien d'une refondation globale du rapport à l'autre qu'il s'agit ${ }^{16}$.

Ce passage de Dacos se conclut de manière ambitieuse par un appel à la « refonte globale du rapport à l'autre », mais la récente évolution du milieu de recherche des digital humanities montre qu'il vise juste. Son article a été rédigé sur la base d'une enquête conduite en 2012 dans ce milieu, alors que la « confiance globish» ne faisait que commencer à réclamer un droit à l'existence. La situation est toutefois en train d'évoluer peu à peu vers une diversification linguistique dans la recherche internationale, comme l'a montré la conférence inaugurale donnée en français par Marin Dacos, précisément, au colloque DH2017 à Montréal ${ }^{17}$. Le chapitre 2 reviendra sur ce rapport entre l'anglais et le français au sein de ce domaine de recherche.

Le phénomène de l'hybridité linguistique manifestée dans le globish n'est en aucun cas une innovation dans la longue histoire de l'Occident. A commencer par le Nouveau Testament qui a tout entier été rédigé dans le globish de son temps, le grec dit de la koinè, un grec hybride, mélangé, qui incorpore également de nombreux sémitismes, produits par des personnes lisant les Ecritures de la Bible hébraïque. Tout comme le grec de la koinè était né de l'expansion

15 M. Dacos, « La stratégie du sauna finlandais : Les frontières des Digital Humanities », Digital Studies/Le champ numérique (2016), DoI : <http://doi.org/10.16995/dscn.41>.

16 Dacos, «La stratégie du sauna finlandais », DOI : <http://doi.org/10.16995/dscn.41>.

17 DH2017, <https://dh2017.adho.org/program/keynotes/>. 
des territoires et des conquêtes militaires provoquant le mélange des cultures ${ }^{18}$, la naissance du globish aura été favorisée par notre propension à voyager et à accaparer de nouveaux territoires, y compris numériques. C'est bien cette capacité à repenser les configurations géographiques et les liens qui est en jeu au cœur de notre pensée duale français/anglais, ou français/globish. On cherche à lier nos mots avec les mots des autres, autour des choses, pour reprendre une thématique foucaldienne bien connue ${ }^{19}$.

De fait, je scrute l'asymétrie linguistique depuis un premier article portant sur l'arrivée de l'ère digitale, intitulé en français «L'ère d'après », mais en en anglais « Common Era $2.0 »^{20}$. Les deux expressions diffèrent à plus d'un titre. L'anglais se faisait plus conquérant ou affirmatif, le français plus prudent et elliptique. Pour dire la nouveauté, l'anglais augmente le présent qu'il désigne comme Common Era, tandis que le français fait la comparaison avec l'ère présente, sans toutefois la nommer. Là où l'anglais explicite et parie sur le futur pour augmenter le présent, le français garde la référence au passé et reste implicite pour désigner l'après.

Qu'on prenne cet exemple ou les titre et sous-titre de ce volume, voici donc le français accompagné d'une langue seconde, l'anglais. Avec cet acolyte, il risque de devenir aussi une langue secondée. Au français de montrer qu'il peut subvertir ce qui lui arrive lorsque l'anglais réclame sa place. Convaincue, comme Marin Dacos, qu'il se joue là quelque chose d'important, et qu'il nous faut le conscientiser et agir, j'ai fait les choix suivants dans ce volume : outre le résumé en anglais de chaque chapitre, une terminologie sera illustrée dans les deux langues à chaque fois que cela sera nécessaire, comme par exemple le terme « esprit », rendu tantôt par mind, tantôt par spirit en anglais ${ }^{21}$. J'espère ainsi contribuer au débat ouvert autour des langues de la recherche.

Cet ouvrage fait aussi le pari que le français peut gagner à cet exercice, où il pourrait sembler perdre de son autonomie et capituler au pouvoir du dominant linguistique. Il espère également que l'anglais ressortira enrichi de ce regard d'ailleurs. C'est à dessein que j'ai rédigé en anglais la version originale de cet article paru en 2017, "Lost in translation? The odyssey of 'digital

18 Voir par exemple U. Eco, «Interprétation et histoire », dans Interprétation et surinterprétation (Formes sémiotiques), S. Collini (éd.), J.-P. Cometti (trad.), PUF, 1996, p. 21-39.

19 M. Foucault, Les mots et les choses, Gallimard, 1966.

20 C. Clivaz, « L'ère d'après ou Common Era 2.o. Lire la culture digitale depuis l'antiquité et la modernité », dans C. Clivaz - J. Meizoz - F. Vallotton - J. Verheyden (éd.), avec B. Bertho, Reading Tomorrow. From Ancient Manuscripts to the Digital Era / Lire Demain. Des manuscrits antiques à l'ère digitale, PPUR, 2012, papier et ebook, p. 3-22 ; Clivaz, « Common Era $2.0 »$.

Voir chapitre 2, partie 2. 
humanities' in French $»^{22}$. C'était tenter de dire à d'autres ce qui se passe dans ma langue qui hérite de l'expression digital humanities, en la réinterprétant et en se la réappropriant. La matière de cet article se trouve traduite en français et répartie dans les chapitres 2 et 3 . Dans le geste de sa traduction, j'y tente l'exercice inverse : nous expliciter à nous-même un peu de ce qu'il advient au français lorsque cette langue choisit de dédoubler l'expression digital humanities par « humanités numériques » ou « humanités digitales».

Voir le français accolé à l'anglais est bien sûr un trait qui tend à s'étendre à l'ensemble des sciences humaines, celles qui sont réflexives en particulier, par le simple fait que nous nous habituons chaque jour davantage à entendre cette autre langue et à la parler. J'en prendrai pour exemple un ouvrage récent de la philosophe Isabelle Stengers, Civiliser la modernité ?, où l'anglais joue un rôle capital, bien que non thématisé comme tel ${ }^{23}$. D'abord parce qu'elle y discute Whitehead, mais aussi parce que nombre de ses interlocuteurs sont anglophones. L'un des points-clés, l'acronyme «SF » qui commande l'architecture de la visée de l'ouvrage ${ }^{24}$, ne se laisse décliner qu'en anglais dans la pluralité de ses significations. Il s'agit, dit-elle, d'un «genre que Donna Haraway a nommé $\mathrm{SF}$, un genre qui démultiplie les sens que peut prendre cet acronyme : sciencefiction, mais aussi speculative fabulation, scientific fact, speculative feminism, string figures, so far... $»^{25}$.

Le français devient ici secondé par cette langue qu'il pensait pouvoir garder secondaire et sans reprendre à son compte, dans ses mots, cette polyvalence. Il $\mathrm{y}$ a pourtant fort à parier qu'il arrive quelque chose à l'expression string figure quand Stengers la traduit par «jeu de ficelle », sans qu'elle ne s'attarde toutefois sur le décalage existant entre figure et «jeu». La philosophe est ici éminemment représentative de ce phénomène d'« interlangue » qui se développe en sciences humaines dans leur rapport désormais inévitable à l'anglais, souvent non explicité. Pour ma part, je montrerai ce qu'il arrive à la langue lors de l'appropriation de l'expression digital humanities en français, au chapitre 2.

En résumé de ces deux premiers points introductifs, on constate que la migration de l'écriture vers la culture digitale sollicite notamment les thématiques du corps, de la langue et des mots. Ces thématiques sont amplifiées, densifiées et complexifiées dans ce qui advient à l'écriture multilingue,

22 C. Clivaz, « Lost in translation? The odyssey of 'digital humanities' in French », Studia UBв Digitalia 62 (2017/1), p. 26-41; <https://digihubb.centre.ubbcluj.ro/journal/index.php/ digitalia/article/view/4>.

25 Stengers, Civiliser la modernité ?, édition Kindle, 1. 2775-2777. 
sur internet et le support digital ${ }^{26}$. En conséquence, le chapitre 3 déploiera l'enquête sur ce qui advient à l'écriture et à son rôle dans la communication d'une culture multimodale digitale. Dans cette culture, le texte devient un vecteur d'information parmi d'autres ${ }^{27}$, une évolution qui ne peut manquer d'influencer la perception des «Ecritures », le donné biblique de la tradition judéo-chrétienne ${ }^{28}$. C'est ce deuxième pan de l'enquête, conduit dans les chapitres 1 et 4, que balise le troisième point de cette introduction.

\section{$4 \quad$ La Bible au creuset de la culture digitale}

C'est une litote de dire que la place dévolue dans les pays européens à la formation aux cultures antiques diminue, à commencer par l'enseignement du grec ancien dans les années qui précèdent l'entrée à l'université. Pour prendre un exemple en Suisse, le grec ancien a récemment cessé d'être enseigné dans les lycées du canton de Berne, en toute discrétion. Dans la présentation du plan d'études concerné, une phrase indique sobrement que «pour des raisons financières, les options spécifiques grec et russe ne sont pas proposées actuellement ${ }^{29}$. Le temps culturel semble bien révolu où Eric Auerbach pouvait ouvrir Mimesis, à Istanbul en 1945, en tablant sur la connaissance que ses lecteurs avaient de l'œuvre homérique : « Les lecteurs de l'Odyssée se souviennent de la scène émouvante et longuement préparée du chant 19 où Ulysse, de retour à Ithaque, est reconnu par sa vieille nourrice Euryclée à la cicatrice qu'il porte à la cuisse $»^{30}$. Qui, même dans le lectorat cultivé et universitaire, se souvient en-

26 Voir par exemple les développements de la traduction automatique: Fonds National Suisse, «Traduction automatique : au-delà du phrase par phrase», 3 avril 2017, <http:// www.snf.ch/fr/pointrecherche/newsroom/Pages/news-170403-communique-de-pressetraduction-automatique-au-dela-du-phrase-par-phrase.aspx $>$.

27 Voir le chapitre 3, et en amont C. Clivaz, «Multimodal Literacies and Continuous Data Publishing : une question de rythme », dans Advances in Digital Scholarly Editing. Papers presented at the DiXiT conferences in The Hague, P. Boot, A. Cappellotto, W. Dillen, F. Fischer, A. Kelly, M. Aodhán, A. Mertgens, A.-M. Sichani, E. Spadini, D. van Hulle (éd.), Sidestone Press, 2017, p. 87-92.

28 Voir le constat dressé en 2011 : «Après deux générations complexes de transition, des années 1940 à l'an 2000, une nouvelle ère culturelle s'annonce, inaugurée par la décade 20012011, et que je propose de nommer 2.o, 'l'ère d'après', puisqu'elle s'enchaîne à cette ère dite 'chrétienne', une expression à propos de laquelle cet article se questionne » (C. Clivaz, «L'ère d'après ou Common Era 2.o », p. 3).

29 Gymnase. Filière de formation préparant aux études supérieures. Direction de l'instruction publique du canton de Berne, $\mathrm{n}^{\circ} 629$ (2017), p. 1-16, ici p. 6, <https://tinyurl.com/ydhlr3dg>.

30 E. Auerbach, Mimesis. La représentation de la réalité dans la littérature occidentale, C. Heim (trad.), Gallimard, 1968, p. 11. 
core d'Euryclée et de la cicatrice d'Ulysse, cette scène-clé qui a servi de matrice à tant de récits de reconnaissance dans l'Antiquité ? Le recul de la culture antique dans son ensemble apparaît aujourd'hui presqu'aussi irrémédiable que la fonte de la banquise, une remarque qui s'inscrit dans un pessimisme culturel manifesté par bien des intellectuels, par exemple Isabelle Stengers :

Cette « civilisation» est marquée par la destruction de ces dispositifs qu'ont cultivés des traditions pour qui le langage n'ouvrait pas à l'hégémonie du désert mais participait à la fabrique sans cesse à reprendre de mondes toujours précaires. On pourrait dire qu'à la question du déclin de notre civilisation a succédé celle d'une débâcle. Nous entendons déjà les grincements et les craquements sourds marquant la rupture des plaques de glace, démantibulant le sol que nous avions défini comme assuré 31 .

Sur cet arrière-plan culturel, que dire alors de la perception de la Bible, et du Nouveau Testament en particulier? Nous arrivons encore à concevoir qu'en 1927, l'exégète Henry Cadbury écrivît qu'« un essai qui éclaire de manière nouvelle une partie du Nouveau Testament ne requiert pas d'apologie », et que cet ouvrage s'imposait « comme best-seller » au sens premier du terme. Mais on s'étonne qu'en 1979 l'exégète Willem van Unnik pût encore paisiblement écrire que la littérature évangélique concernait « toute la terre, toutes les nations, [... et] les hommes placés devant la dernière décision de vie ou de mort éternelles ${ }^{32}$. L'évolution de la sociologie des chrétiens en Europe, - et en corollaire celle de la population académique de la théologie chrétienne - devrait inviter tout exégète à renoncer désormais à une telle universalisation.

De fait, le bouleversement de la place accordée dans nos sociétés à la formation en théologie chrétienne a suscité des débats souvent vigoureux dans divers lieux institutionnels ${ }^{33}$. Dans ce débat, la transformation du support d'écriture, et ce qu'elle implique pour le christianisme et les Ecritures bibliques, n’a guère été prise en compte. Pourtant elle est un lieu clé du rapport du christianisme à notre contexte culturel contemporain : il n'est pas de meilleur chemin pour se prémunir de donner d'entrée à la Bible un statut extrinsèque, qui pourrait encore lui être attribué selon la métaphore du «grand angle »

$31 \quad$ Stengers, Civiliser la modernité ?, édition Kindle, 1. 3176.

32 W. C. van Unnik, « Luke's Second Book and the Rules of Hellenistic Historiography », dans J. Kremer (éd.), Les Actes des Apôtres. Traditions, rédaction, théologie (BEThL 48), Leuven University Press/J. Duculot, 1979, p. 37-6o ; ici p. 49.

33 Voir par exemple P. Gisel, Traiter du religieux à l'université. Une dispute socialement révélatrice (coll. Contre-pied), 2011. 
évoquée par Carrière ${ }^{34}$. Si phénomène de grand angle il y a ou il y a eu, c'est précisément sous l'influence de la culture imprimée qui, au plus fort de son impact, donne naissance à l'expression des «religions du Livre». En 2012, j'avais en effet signalé que cette expression - à distinguer des ahl al-Kitab, les gens du Livre mentionnés dans le Coran - se développe au moment de l'apogée de la culture imprimée, dans la seconde moitié du $19^{\mathrm{e}}$ siècle, autour d'une série de conférences données par Friedrich Max Müller ${ }^{35}$. La connivence entre culture du grand angle des religions du Livre et culture imprimée sera discutée au chapitre $4^{36}$, et s'inscrira dans une analyse globale de la transformation culturelle en cours.

Grand angle oblige, l'héritage chrétien est au cœur de ce qui se défait et se recompose. Depuis le début du $21^{\mathrm{e}}$ siècle, Edward Said en tête, plusieurs voix ont souligné qu'Auerbach avait encore choisi l'ancre de la téléologie chrétienne en rédigeant Mimesis, rangeant Homère du côté de la fiction, et la Bible du côté de l'histoire ${ }^{37}$. Or les années de fin de guerre et d'après-guerre sont précisément celles où se prépare la rencontre de l'informatique et des sciences humaines, sous l'égide de la figure quasi tutélaire du jésuite Roberto Busa : il vaut la peine de s'arrêter à cette interaction et à la généalogie - ou aux généalogies diversifiées - qu'on peut maintenant attribuer à l'émergence de ces humanités en voie de digitalisation ${ }^{38}$. Quant à la première décade du $21^{\mathrm{e}}$ siècle, celle où le contexte général de la pensée d'Auerbach devient plus perceptible, elle est aussi celle de la naissance de l'appellation digital humanities ${ }^{39}$. Il fait donc sens

34 Voir page 2 ci-dessus.

35 C. Clivaz, «Homer and the New Testament as 'Multitexts' in the Digital Age », Scholarly and Research Communication 3 (2012/3), p. 1-15; <http://src-online.ca/index.php/src/arti cle/view/97> ; ici p. 4 ; F. M. Müller, « Second Lecture Delivered at the Royal Institution, February 26, 1870 », dans Introduction to the science of religion. Four lectures delivered at the Royal Institution, F. M. Müller, Wilson Press, 2010, p. 52-82.

$36 \quad$ Voir chapitre 4, point 2.3.1.

37 E. Said, «Introduction», dans E. Said, Mimesis : the Representation of Reality in Western Literature, W. S. Trask (trad.), Princeton University Press, 2003, p. IX-XXXII ; ici p. IX : « Auerbach prétend que le vrai concept de figura fonctionne aussi comme un moyen terme entre la dimension littéraire-historique et, pour l'auteur chrétien, le monde de la vérité, veritas ». Partagent notamment cette lecture Stephen Greenblatt, Catherine Gallagher et Richard Walsh ; voir C. Clivaz, L'ange et la sueur de sang (Lc 22,43-44), ou comment on pourrait bien encore écrire l'histoire (BiTS 7 ), Peeters, 2010, p. 10-11.

38 Voir notamment S. E. Jones, Roberto Busa, S.J., and the emergence of Humanities Computing. The priest and the punched cards, Routledge, 2016; J. Nyhan - A. Flinn, Computation and the Humanities Towards an Oral History of Digital Humanities (Spring series on Cultural Computing), Spring, 2016.

Voir Clivaz, « Common Era 2.0 », p. 18-21. 
de considérer cette période de 1945 à nos jours, pour y discerner ce qui s'y lie et s'y délie, entre héritage chrétien, sciences humaines et informatique.

Une telle analyse apparaît d'autant plus nécessaire que la seule monographie existant à ma connaissance à ce jour sur la Bible dans la culture digitale, s'adresse délibérément à un public christianisé et chrétien, sous la plume de Jeffrey Siker (Liquid Scripture, 2017) ${ }^{40}$. Le passage suivant n'a rien à envier à la posture de van Unnik en 1979 : « La Bible [...] n'est pas un simple livre assemblé, mais le Livre de tous les livres. Aucune surprise à voir la Bible continuer à être le livre le mieux vendu de tous les temps, année après année, version après version, traduction après traduction ${ }^{41}$. Au contraire de cette approche qui postule encore - comme c'est souvent le cas aux Etats-Unis - un contexte culturel chrétien très présent, je propose de situer ce qu'il advient des Ecritures en lisant d'abord ce qu'il advient de l'écriture en général, quitte bien sûr à mesurer ensuite l'écart créé par l'« effet grand angle » biblique. Tel est donc le programme de notre parcours : ce qui arrive à l'écriture - via la langue, les mots et le corps - lorsqu'elle s'aventure sur le support d'écriture digital (chapitres 2 et 3), et ce qui arrive aussi aux Ecritures bibliques digitalisées (chapitres 1 et 4).

40 J. S. Siker, Liquid Scripture. The Bible in a Digital World, Fortres Press, 2017 ; C. Clivaz, « Review of Jeffrey S. Siker, Liquid Scripture : The Bible in a Digital World », RBL 05 (2018), p. 1-6, <https://www.bookreviews.org/pdf/11851_13221.pdf>.

41 Siker, Liquid Scriptures, p. 9 : « The Bible [...is] not just a unified book, but the Book of all books. It is no wonder that the Bible continues to be the best-selling book of all time, year after year, version after version, translation after translation ». 